\title{
O impacto de uma era pandêmica e isolamento social na saúde mental: uma revisão
}

\section{integrativa}

\author{
The impact of a pandemic era and social isolation on mental health: a literature review \\ El impacto de una era pandémica y el aislamiento social en la salud mental: una revisión de la
}

\section{literatura}

Recebido: 12/01/2021 | Revisado: 14/01/2021 | Aceito: 18/01/2021 | Publicado: 21/01/2021

Thayná Brenda Benicio Ferreira Bastos

ORCID: https://orcid.org/0000-0003-1495-5345

Faculdade Estácio Teresina, Brasil

E-mail: thyabenicio16@gmail.com

Thaysla de Oliveira Sousa

ORCID: https://orcid.org/0000-0001-9192-1099

Faculdade Estácio Teresina, Brasil

E-mail: thayslalaurentina25@gmail.com

Aleissane Kananda Lacerta Torres

ORCID: https://orcid.org/0000-0002-0860-8550

Faculdade Estácio Teresina, Brasil

E-mail: aleissane-kananda@hotmail.com

Hyago Arouche Cardoso

ORCID: https://orcid.org/0000-0002-3101-374X

Faculdade Estácio Teresina, Brasil

E-mail: hyagoarouche98@gmail.com

Anderson Lima Dos Santos

ORCID: https://orcid.org/0000-0002-3292-4860

Faculdade Estácio Teresina, Brasil

E-mail: andersonb124@hotmail.com

Cidianna Emanuelly Melo do Nascimento ORCID: https://orcid.org/0000-0001-5477-4413

Universidade Estadual do Ceará, Brasil

E-mail: profa.cidianna.melo@gmail.com

Filipe Augusto de Freitas Soares

ORCID: https://orcid.org/0000-0003-0510-3968

Faculdade Estácio de Teresina, Brasil

E-mail: filipe-freitas_2008@hotmail.com

\begin{abstract}
Resumo
Introdução: Por conta do aumento simultâneo de casos, óbitos e países afetados pelo novo coronavírus, a Organização Mundial da Saúde (OMS) declarou que o episódio constituía uma emergência de saúde pública de importância mundial. Para suavizar os impactos da pandemia, alguns países adotaram o isolamento de casos suspeitos, distanciamento social, e quarentena para toda a população. Afora os impactos psicológicos que estão diretamente relacionados à COVID-19 principalmente quando se trata de isolamento social, medidas para restrição da pandemia também podem consistir em fatores de risco à saúde mental. Objetivo: Descrever os impactos na saúde mental em meio ao isolamento social. Metodologia: Este estudo trata-se de uma revisão de literatura realizada no período de julho de 2020. A busca das publicações ocorreu via Biblioteca Virtual de Saúde, no período de julho de 2020. Foram utilizados os critérios de inclusão: artigos com texto completo nos idiomas português inglês e espanhol e publicados no ano de 2020 e de exclusão: artigos repetidos e que não respondem á questão norteadora. Resultado e discussão: Dos 72 artigos analisados 8 foram selecionados para uma análise minuciosa do estudo, após aplicação dos critérios de inclusão e exclusão. Percebeu-se que, inúmeros são os efeitos psicológicos negativos causados pela pandemia do Covid-19. Incerteza do fim da quarentena, tédio por conta do confinamento, medo de adquirir a infecção, perda financeira contribuem para o aumento do número de casos de pessoas que desenvolveram ou agravaram doenças mentais. Conclusão: Conclui-se a importância de criar estratégias de ação em promoção da saúde mental para a população como um todo, uma vez que todos de alguma forma estão vivenciando um momento marcado pela tensão, medo, insegurança e ansiedade.
\end{abstract}

Palavras-chave: Coronavírus; Saúde mental; Isolamento social; Pandemia. 


\begin{abstract}
Introduction: Due to the simultaneous increase in cases, deaths and countries affected by the new coronavirus, the World Health Organization (WHO) declared that the episode constituted a public health emergency of global importance. To mitigate the impacts of the pandemic, some countries have adopted the isolation of suspected cases, social distance, and quarantine for the entire population. Aparte de los impactos psicológicos que están directamente relacionados con COVID-19, especialmente cuando se trata de aislamiento social, las medidas para restringir la pandemia también pueden consistir en factores de riesgo para la salud mental. Objective: to describe the impacts on mental health in the midst of social isolation. Methodology: This study is a literature review carried out in the period of July 2020. The search for publications occurred via the Virtual Health Library, in the period of July 2020. Inclusion criteria were used: articles with full text in Portuguese, English and Spanish languages and published in the year 2020 and exclusion: repeated articles that do not answer the guiding question. Result and discussion: Of the 72 articles analyzed, 8 were selected for a thorough analysis of the study, after applying the inclusion and exclusion criteria. it was realized that there are countless negative psychological effects caused by the pandemic of the Covid-19. Uncertainty at the end of the quarantine, boredom due to confinement, fear of acquiring the infection, financial loss contribute to the increase in the number of cases of people who developed or worsened mental illnesses. Conclusion: We conclude the importance of creating action strategies to promote mental health for the population as a whole, since everyone is somehow experiencing a moment marked by tension, fear, insecurity and anxiety.
\end{abstract}

Keywords: Coronavirus; Mental health; Social isolation; Pandemic.

\title{
Resumen
}

Introducción: Debido al aumento simultáneo de casos, muertes y países afectados por el nuevo coronavirus, la Organización Mundial de la Salud (OMS) declaró que el episódio constituyó una emergencia de salud pública de importância mundial. Para mitigar los impactos de la pandemia, algunos países han adoptado el aislamiento de casos sospechosos, la distancia social y la cuarentena para toda la población. Aside from the psychological impacts that are directly related to COVID-19, especially when it comes to social isolation, measures to restrict the pandemic may also consist of risk factors for mental health. Objetivo: describir los impactos sobre la salud mental en medio del aislamiento social. Metodología: Este estudio es una revisión de la literatura realizada en el período de julio de 2020. La búsqueda de publicaciones se realizó a través de la Biblioteca Virtual en Salud, en el período de julio de 2020. Se utilizaron criterios de inclusión: artículos con texto completo en Idiomas portugués, inglés y español y publicados en el año 2020 y exclusión: artículos repetidos que no responden a la pregunta orientadora. Resultado y discusión: De los 72 artículos analizados, 8 fueron seleccionados para un análisis exhaustivo del estudio, luego de aplicar los criterios de inclusión y exclusión. Se advirtió que existen innumerables efectos psicológicos negativos provocados por la pandemia del Covid-19. La in certidumbre al final de la cuarentena, el aburrimiento debido al confinamiento, el miedo a adquirir la infección, la pérdida económica contribuyen al aumento de casos de personas que desarrollaron o empeoraron enfermedades mentales. Conclusión: Concluimos la importancia de crear estrategias de acción para promover la salud mental de la población en su conjunto, ya que todos de alguna manera estamos viviendo un momento marcado por la tensión, el miedo, la inseguridad y la ansiedad.

Palabras clave: Coronavirus; Mental health; Social isolation; Pandemic.

\section{Introdução}

No mês de dezembro de 2019, na cidade de Wuham, província de Hubei, na China, surgiu o COVID-19, que foi identificado como agente etiológico de um surto de pneumonias. Por conta do aumento simultâneo de casos, óbitos e países afetados a Organização Mundial da Saúde (OMS) declarou que o episódio constituía uma emergência de saúde pública de importância mundial. O Brasil, em 3 de fevereiro de 2020, também declarou a pandemia como emergência em saúde pública de importância internacional (Martins, 2020).

Com o propósito de suavizar os impactos da pandemia, reduzir o pico de incidência e o número de mortes, alguns países têm adotado algumas medidas, tais como isolamento de casos suspeitos, fechamento de escolas e universidades, distanciamento social de idosos e outros grupos de risco, bem como quarentena de toda a população (Brooks, 2020; Fergunson, 2020).

Afora os impactos psicológicos que estão diretamente relacionados à COVID-19 principalmente quando se trata de isolamento social, medidas para restrição da pandemia também podem consistir em fatores de risco à saúde mental. Em revisão de literatura sobre a quarentena, Brooks (2020) identificaram que os efeitos de rejeição dessa medida incluem sintomas de estresse pós-traumático, confusão e raiva. Preocupações com a diminuição suprimentos e as perdas financeiras também 
acarretam prejuízos ao bem-estar psicológico (Shojaei \& Masoumi, in press, 2020).

O surto de COVID-19 é emocionalmente desafiador para todos, especialmente, para indivíduos que sofrem de depressão, e de outras doenças mentais que necessitam de uma atenção psicossocial. Podendo afetar cada um de maneira diferente, dependendo de fatores como história de vida, das suas características e da comunidade em que vive e reação de muitas famílias frente a crise econômica e aumente do desemprego (Klamek, 2020).

É imprescindível que os profissionais da saúde, que atuam constantemente na linha de frente, estejam mais vulneráveis quando se trata da saúde emocional, pois lidam também com seus sentimentos de capacidade, fracasso, estresse pelas condições encontradas e sobrecarga de trabalho, incertezas sobre a doença e tratamento por ser uma doença sem muitos conhecimentos, medo de contrair e transmitir o vírus e até mesmo dificuldade de lidar com perdas de seus pacientes. Relacionam-se também com os familiares acompanhantes dos clientes, suas perdas e tudo que se trata da pandemia como um todo (Xiang, 2020).

Nos encontramos em um momento delicado de pandemia e crise mundial ocasionado pela COVID-19, em que a proteção dos sujeitos está diretamente relacionada ao isolamento social, nos vemos com enormes desafios a serem enfrentados e adaptações a serem realizadas. A necessidade de acolhimento universal tem sido ainda maior em meio a pandemia. Nesse momento ter o conhecimento de como a rede está funcionando e canais de comunicação é extremamente necessário, para que uma escuta consoladora à angústia (Martins, 2020).

No entanto ao observar o comportamento das pessoas em meio ao isolamento social, percebeu-se que os índices de suicídio e doenças mentais vem aumentando. Nesse caso torna-se relevante as pesquisas sobre as consequências do isolamento social devido a COVID-19. Tendo em vista que a adaptação e ansiedade nesse período de quarentena essa pesquisa foi desenvolvida como o objetivo de descrever sobre os impactos na saúde mental em meio a pandemia do COVID-19.

\section{Metodologia}

Trata-se de um estudo com coleta de dados do tipo qualitativa realizada a partir de fontes secundárias, por meio de levantamento bibliográfico e baseado na experiência vivenciada pelas autoras por ocasião da realização de uma revisão integrativa.

A pesquisa bibliográfica é uma das melhores formas de iniciar um estudo, buscando-se semelhanças e diferenças entre os artigos levantados nos documentos de referência. A compilação de informações em meios eletrônicos é um grande avanço para os pesquisadores, democratizando o acesso e proporcionando atualização frequente. A revisão integrativa, finalmente, é a mais ampla abordagem metodológica referente às revisões, permitindo a inclusão de estudos experimentais e nãoexperimentais para uma compreensão completa do fenômeno analisado. Combina também dados da literatura teórica e empírica, além de incorporar um vasto leque de propósitos: definição de conceitos, revisão de teorias e evidências, e análise de problemas metodológicos de um tópico particular. A ampla amostra, em conjunto com a multiplicidade de propostas, deve gerar um panorama consistente e compreensível de conceitos complexos, teorias ou problemas de saúde relevantes para a enfermagem. (Whittemore \& Knafl K 2005)

Este estudo trata-se de uma revisão de in realizada no período de julho de 2020 com base em artigos selecionados em que se destacou os impactos na saúde mental em meio a pandemia do covid-19. Os dados coletados estavam presentes em meios eletrônicos de base de dados como a Biblioteca Virtual de Saúde (BVS-BIREME) e Scientific Electronic Library Online (SCIELO), através dos descritores "coronavírus", "saúde mental", "isolamento social", "pandemia", usado em forma única ou combinada.

Os critérios de inclusão adotados foram: artigos em português inglês e espanhol que disponibilizavam o texto 
completo em suporte eletrônico. Já como critérios de exclusão: teses, livros, anais de congressos ou conferências e relatórios. A amostra deu-se a partir da leitura do resumo dos artigos encontrados e seleção daqueles que responderam ao problema da pesquisa, totalizando 72 artigos. Para mapeamento das produções cientificas utilizou-se uma ficha documental constituída das seguintes variáveis: ano da publicação, nome dos autores e título. Para análise de conteúdo efetuou-se leitura integral e exaustiva dos artigos, para transcrição dos resultados e trechos significativos.

\section{Resultados e Discussão}

Após aplicação dos critérios de inclusão foi feito uma análise minuciosa de 6 artigos, 1 cartilha e 1 revisão bibliográfica sendo estrangeiros e nacionais que abordassem a temática "impacto da pandemia na saúde mental". 
Research, Society and Development, v. 10, n. 1, e41910111905, 2021

(CC BY 4.0) | ISSN 2525-3409 | DOI: http://dx.doi.org/10.33448/rsd-v10i1.11905

Quadro 1. Síntese dos estudos relacionados aos impactos da pandemia do COVID-19 na saúde mental

\begin{tabular}{|c|c|c|c|c|}
\hline Autor (a) & Periódico & País & Objetivos & Conclusão \\
\hline $\begin{array}{l}\text { Brooks K, et.al. } \\
2020 .\end{array}$ & $\begin{array}{lr}\text { Department } & \text { of } \\
\text { Psychological } & \text { Medicine, } \\
\text { King's College } & \end{array}$ & Londres & $\begin{array}{l}\text { Em geral podem ser } \\
\text { Favoráveis mostrar as evidências sobre o impacto } \\
\text { psicológico da quarentena, explorando seus efeitos } \\
\text { sobre a saúde mental e bem-estar psicológico, os } \\
\text { fatores que contribuem para mitigar esses efeitos. }\end{array}$ & $\begin{array}{l}\text { A quarentena é considerada necessária e os } \\
\text { gestores devem tornar a mesma o mais tolerável } \\
\text { possível, fornecendo informações sobre os } \\
\text { protocolos, garantindo suprimentos adequados, } \\
\text { oferecendo atividades significativas, lembrando ao } \\
\text { público os benefícios da quarentena para a } \\
\text { sociedade } \\
\text {. }\end{array}$ \\
\hline Khan S, et al. 2020. & $\begin{array}{lr}\text { Electronic } & \text { supplementary } \\
\text { material: The online } \\
\text { version of this article } \\
\text { contains supplementary } \\
\text { material. }\end{array}$ & China & $\begin{array}{l}\text { Foca nos riscos de distúrbios psiquiátricos associados } \\
\text { ao novo surto de Covid- } 19 \text {. } \\
\text { Criar uma resiliência psicológica entre profissionais } \\
\text { de saúde, o público e as consequências de estarmos } \\
\text { estressados. }\end{array}$ & $\begin{array}{l}\text { Estratégias para abordar a saúde psicológica } \\
\text { causada pela exposição direta ou indireta da } \\
\text { situação, é necessário. Sendo essas, específicas } \\
\text { para atingir toda a população. A resiliência } \\
\text { psicológica pode ser uma estratégia eficaz, o } \\
\text { interrogatório psicológico para pessoas que sofrem } \\
\text { de estresse é recomendado, uma breve terapia } \\
\text { cognitivo-comportamental para pessoas com } \\
\text { sintomas graves de estresses. }\end{array}$ \\
\hline $\begin{array}{l}\text { Marpinko D, et al } \\
2020 \text {. }\end{array}$ & Psychiatria Danubina & Croácia & $\begin{array}{l}\text { Compreender como a crise influencia as reações de } \\
\text { um indivíduo a eventos estressantes, relevando falhas } \\
\text { de trabalhos relacionados à abordagem psicodinâmica } \\
\text { da crise recente. }\end{array}$ & $\begin{array}{l}\text { É importante compreender como a crise influencia } \\
\text { as reações de um indivíduo a eventos estressantes, } \\
\text { se torna extremamente necessário criar estratégias } \\
\text { de intervenções significativas e eficazes. Acredita- } \\
\text { se que a inclusão da abordagem psicodinâmica no } \\
\text { sistema nacional de emergência de saúde pública e } \\
\text { mental capacitará mundo durante (e depois) a crise } \\
\text { de pandemia de Covid-19. }\end{array}$ \\
\hline Gref P, et al. 2020. & Fundação Oswaldo Cruz & Brasil & $\begin{array}{l}\text { Diminuir as tentativas de suicídio meio a pandemia do } \\
\text { Covid-19, abordando atitudes que devem ou não serem } \\
\text { tomadas pela população visando a saúde mental e o } \\
\text { impacto que tais atitudes podem vir a ser. }\end{array}$ & $\begin{array}{l}\text { É necessário aumentar conscientização, diminuir o } \\
\text { estigma e prevenir o suicídio criando campanhas, no } \\
\text { ato da conversa, se capacitando. }\end{array}$ \\
\hline
\end{tabular}


Research, Society and Development, v. 10, n. 1, e41910111905, 2021

(CC BY 4.0) | ISSN 2525-3409 | DOI: http://dx.doi.org/10.33448/rsd-v10i1.11905

\begin{tabular}{|c|c|c|c|c|}
\hline $\begin{array}{l}\text { Fiorillo A, et al. } \\
2020 .\end{array}$ & European Psychiatry & Paris & $\begin{array}{l}\text { Destaca o aumento de casos psíquicos devido ao } \\
\text { isolamento social meio a pandemia de Covid-19, a } \\
\text { pandemia não tem afetado apenas a saúde física mas } \\
\text { também a saúde mental de toda a população. }\end{array}$ & $\begin{array}{l}\text { Aumento de problemas mentais, distúrbios } \\
\text { comportamentais e transtornos de uso de } \\
\text { substâncias, como estressores extremos podem } \\
\text { exacerbar ou induzir problemas psiquiátricos. Uma } \\
\text { grande chance que haja uma escassez de } \\
\text { profissionais de saúde devido ao esgotamento e ao } \\
\text { esgotamento mental. O risco de notícias falsas } \\
\text { correndo mais rápido do que o próprio vírus, e } \\
\text { criando incertezas e preocupações. }\end{array}$ \\
\hline $\begin{array}{l}\text { Depierro L, et al. } \\
2020 .\end{array}$ & Psychiatry Research & Estados Unidos & $\begin{array}{l}\text { Medidas efetivas de prevenção, prováveis necessidades } \\
\text { de tratamento a curto e }\end{array}$ & $\begin{array}{l}\text { Os impactos da pandemia Covid-19 provavelmente } \\
\text { serão sentidos por muitas décadas, assim como o } \\
\text { impacto do } 11 \text { de setembro ainda é sentido por } \\
\text { muitos. Perda de vidas levará a problemas de saúde } \\
\text { mental e impactos na população, incluindo } \\
\text { familiares de vítimas, trabalhadores da saúde que } \\
\text { tentaram valentemente salvar os doentes e outros } \\
\text { funcionários essenciais. }\end{array}$ \\
\hline Cao W, et al. 2020. & Psychiatry Research & China & $\begin{array}{l}\text { Avaliar a condição psicológica de estudantes } \\
\text { universitários durante uma pandemia e explorar fatores } \\
\text { que influencia sua ansiedade }\end{array}$ & $\begin{array}{l}\text { A saúde mental de estudantes universitários é } \\
\text { afetada quando confrontada com emergências de } \\
\text { saúde pública e requer atenção, ajuda e apoio da } \\
\text { sociedade, famílias e faculdades. Sugere-se que o } \\
\text { governo e as escolas devem colaborar para resolver } \\
\text { esse problema para fornecer serviços psicológicos } \\
\text { de alta qualidade e oportunos, orientados para } \\
\text { crises, a estudantes universitários. }\end{array}$ \\
\hline
\end{tabular}

Fonte: Autores. 
Uma forma importante de minimizar os impactos causados pelo isolamento social, é através de informações, sobre o desenvolvimento de depressão clínica e sintomas relacionados ao suicídio ocasionado por esse momento. Com base nas ideias de Khan et al. (2020) os psicólogos clínicos, psiquiatras e enfermeiros possuem um papel fundamental de se comunicar através dos recursos midiáticos para incentivar as pessoas a viverem atividades saudáveis, transmitindo instruções sobre relaxamento, meditação e conselhos para que as pessoas não se concentrem em notícias falsas de fontes não autênticas.

De acordo com Brooks et al. (2020) alguns estudos mostraram que durações mais longas de quarentena foram associadas especificamente à piora do estado mental, sintomas de estresse pós-traumático, comportamentos de esquiva, e raiva. Embora a duração da quarentena tenha sido sempre bem clara, um estudo mostrou que aqueles que ficaram em quarentena por mais de 10 dias apresentaram significativamente mais sintomas de estresse pós-traumático do que aqueles vivenciaram por menos de 10 dias.

Ainda seguindo a linha de pensamento de Brooks et al. (2020), verificou-se que profissionais de saúde que vivenciaram essa situação apresentaram sintomas mais graves de estresse pós-traumático do que os membros do público em geral, obtendo uma pontuação significativamente maior em todas as dimensões. Observou-se que essas pessoas foram submetidas a bem mais estigmas e preconceitos quando comparado com as demais pessoas, demonstrando mais comportamentos de esquiva após quarentena. Além disso, relataram maior perda de renda, mais raiva, aborrecimento, medo, frustração, culpa, desamparo, isolamento, solidão, nervosismo, tristeza, preocupação e eram menos felizes. Os profissionais de saúde devido a exposição à patologia estavam mais suscetíveis a contrair, assim como pensar que tinham síndrome respiratória aguda grave, gerando uma grande preocupação não só com seu bem-estar e sua saúde, mas também, se está infectando outras pessoas e disseminando a doença.

Outro grupo afetado é retratado no estudo de Cao et al. (2020), onde mostrou que cerca de 24,9\% dos estudantes universitários experimentaram ansiedade por causa deste surto de COVID-19. Foi possível analisar que morar em áreas urbanas ou com os pais e ter uma renda familiar estável foram fatores de proteção para estudantes universitários contra a ansiedade experimentada durante o surto de COVID-19. Todavia, ter um parente ou conhecido infectado com COVID-19 era um fator de risco independente para ansiedade experimentada.

Consoante Fiorillo e Gorwood (2020) um aspecto que deve ser considerado está relacionado ao estigma e à discriminação contra pessoas infectadas e seus familiares. É importante combater o estigma social em relação a pessoas que tratam e cuidam de pessoas com COVIDd-19 devendo ser outra prioridade para os profissionais de saúde mental nos próximos meses.

DePierro, Lowe e Katz (2020) fizeram em seu estudo uma comparação entre o acontecimento de 11 de setembro e a pandemia do COVID-19 e concluíram que a grande perda de vidas levará a problemas de saúde mental e impactos na população, incluindo familiares de vítimas, trabalhadores da saúde que tentaram de todas as formas possíveis salvar os doentes e outros funcionários essenciais que continuaram a servir o público mesmo com a ameaça permanente à vida. Em vez da súbita onda de medo que acompanhou os ataques de 11 de setembro, a pandemia de COVID-19 provavelmente trará uma onda mais insidiosa de ansiedade, raiva e sofrimento conforme os números das vítimas aumentam.

Inko et al. (2020) enfatizou como importante compreender como a crise influencia as reações de um indivíduo a eventos estressantes (e vice-versa) para criar intervenções significativas e eficazes. Nesse contexto, observou que apesar do imenso esforço dos profissionais de saúde para lidar com distúrbios relacionados ao estresse e trauma no curso da pandemia de COVID-19, uma grande proporção de pessoas afetadas pelo transtorno não tem acesso a tratamento psicológico ou psiquiátrico.

Conforme o pensamento Jakovljevic et al. (2020) é necessário reavaliar e melhorar o entendimento básico de como a sociedade humana e o mundo global operam a partir da abordagem científica multidisciplinar e transdisciplinar. As ciências da 
saúde mental poderiam ajudar a progredir na construção de uma sociedade compassiva e de uma civilização empática, que seriam mais eficazes na prevenção e superação de epidemias e outras ameaças globais à humanidade. A promoção da saúde mental global está relacionada à promoção do auto humanização e da civilização empática, assim como a promoção da saúde mental pública está relacionada à promoção da sociedade compassiva.

Por fim, através do embasamento científico de Greff et al. (2020) ressalta-se que a intervenção precoce, particularmente na primeira fase da resposta a uma pandemia, bem como o fortalecimento das redes e laços socioafetivos e o acesso aos tratamentos de saúde mental são considerados fatores protetivos. É esperado que uma pandemia, nas proporções da Covid-19, promova instabilidade psíquica e desencadeie reações que possam potencializar pensamentos e atos suicidas. Nesse sentido, é importante promover estratégias que permitam contrabalançar os sentimentos negativos e reenquadramento dos planos de vida.

\section{Conclusão}

Evidencia-se, após análise dos estudos, que durante uma pandemia, as ações relacionadas à higienização da mão, da residência e o uso constante de máscaras, estão entre as reações comportamentais mais comuns, é esperado que estejamos frequentemente em estado de alerta, preocupados, confusos, estressados e com sensação de falta de controle frente às incertezas do momento, percebeu-se também que inúmeros são os efeitos psicológicos negativos causados pela pandemia da COVID-19.

A incerteza com relação a duração da quarentena, medo de contrair a infecção, tédio devido o confinamento, preocupação com a falta de suprimentos como alimentação, informações inadequadas e exageradas expostas pela mídia, crise financeira, preconceito e tabus relacionados a doença, medo de perder os familiares, luto vazio por não poder velar algum ente querido, nervosismo, tristeza, entre outras causas, contribuem para o aumento do número de casos de pessoas que desenvolveram ou agravaram doenças mentais, tais como: estresse pós traumático, depressão, ansiedade, esquizofrenia, confusão, irritabilidade, baixo humor, distúrbios emocionais e insônia.

É necessário prestar uma assistência de qualidade tanto para os pacientes quanto para seus cuidadores, já que muitas vezes precisam de tratamento a longo prazo, apoio e aconselhamento contínuos. Diante do exposto, foi notório uma escassez de estudos relacionados a essa temática, tendo em vista a sua importância faz se necessário a realização contínua de estudos, relacionados aos impactos mentais do isolamento assim como o compartilhamento de práticas de promoção a saúde mental do meio acadêmico para a população.

\section{Referências}

Brooks, S. K., Rebecca, K. W., Louise, E. S., Lisa, W., Simon, W., \& Neil, G. (2020). The psychological impact of quarantine and how to reduce it: rapid review of the evidence. Department of Psychological Medicine, King's College, 395-10227, Londres.

Cao, W., Ziwei, F., Guoqiang, H., Mei, H., Xinrong, X., Jiaxin, D., \& Jianzhong, Z. (2020). The sychological impact of the COVID-19 epidemic on college students in China. Psychiatry Research, 287- 112934. China.

Depierro, J., Lowe, S., \& Katz, C. (2020) Lessons learned from. Mental health perspectives on the COVID-19 pandemic. Psychiatry Research, 288- 112934, Estados Unidos.

Ferguson N., Laydon, D., Nedjati Gilani, G., Imai, N., Ainslie, K., Baguelin, M., Baguelin, M., Boonyasiri, A., Cucunuba Perez, Z., Cuomo-Dannenburg, G., Dighe, A., Dorigatti, I., Fu, H., Gay Thorpe, K., Green, W., Hamlet, A., Hinsley, W., Okell, L., Van Elsland, S., Thompson, H., Verity, R., Volz, E., Wang, H., Wang, Y., Walker, P., Walters, C., Win skill, P., Whittaker, C., Donnelly, C., Riley, S., \& Ghani, A., (2020). Impact of non-pharmaceutical interventions (INP) to reduce mortality from COVID19. Imperial College COVID-19 Response Team. 10-25561.

Fiorillo, A. \& Gorwood, P. (2020) The consequences of the COVID-19 pandemic on mental health and implications for clinical practice. European Psychiatry, 2020.35.

Greff, A. P., et al. (2020) Saúde Mental e Atenção Psicossocial na Pandemia Covid-19. Fundação Oswaldo Cruz. 41-420. 
Research, Society and Development, v. 10, n. 1, e41910111905, 2021 (CC BY 4.0) | ISSN 2525-3409 | DOI: http://dx.doi.org/10.33448/rsd-v10i1.11905

Jakovljevic, M., Bjedov, S., Jaksic, N., Jakovljevic, I. (2020) Covid-19 pandemia and public and global mental health from the perspective of global health security. Psychiatria Danubina, 32- 1614.

Khan, S., Rabeea, S.., Hongmin, L.., Ashaq, A.., Muhammad, A. S., Nadia, B.., \& Mengzhou, X. (2020). Impact of coronavirus outbreak on psychological health. Electronic supplementary material: The online version of this article contains supplementary material, $10-010331$.

Klamek, A. B., \& Anat, B. (2020) Suicide prevention during the COVID-19 outbreak. The lancet, 32-353271Israel.

Martins, D. S. et al. (2020). Da proximidade ao distanciamento social: desafios de sustentar a lógica da atenção psicossocial em tempos de pandemia. Relato de experiência do Centro de Atenção Psicossocial Infantil - CAPSi Asa Norte no Distrito Federal. Health Residencies Journal, 11 - 2020.

MarPinko, D., Miro, J., Nenad, J., Sarah, B., Aleksandra, M. D. (2020) The importance of psychodynamic approach during covid-19 pandemic. Psychiatria Danubina, 32-11525.

Whittemore, R., \& Knafl, K. The integrative review: update methodology. J Adv Nurs. 2005;52(5):546-53

Chmidt, B., Crepaldi, M. A., Bolze, S. D. A., Neiva,-S. L., \& Demenech, L. M. (2020). Saúde mental e intervenções psicológicas diante da pandemia do novo coronavírus (COVID-19). Estudos de Psicologia (Campinas), 37-200063.

Shojaei, B., Masoum, M. A., Azeredo, S. D., Neiva,-S, L., \& Demenech, L. M. (2020). Mental health and psychological interventions in the face of the new coronavirus pandemic (COVID-19). Psychology Studies 37-200063.

Singhal, T. A (2020) Review of coronavius Disease (COVID-19). Indian J Pediatr 87, 281-286.

Souza, M. T., Silva, M. D., Carvalho, R (2010). Revisão integrativa: o que é e como fazer 81-1026.

Xiang, A., Bahiano, M. de A., Nakano, T. de C., Reis, C., Silva, B. F. Pereira da, \& Vitti, L. Santos. (2020). Covid-19 and mental health: the emergence of care. Psychology Studies 37-200074. 